Central Washington University

ScholarWorks@CWU

All Faculty Scholarship for the College of the Sciences

6-1995

Vibrational properties of a loaded string

Samantha Parmley

Tom Zobrist

Terry Clough

Anthony Perez-Miller

Mark Makela

Follow this and additional works at: https://digitalcommons.cwu.edu/cotsfac

Part of the Quantum Physics Commons 
${ }^{1}$ Paul A. Tipler, Physics for Scientists and Engineers (Worth, New York, 1991), Chap. 11.

${ }^{2}$ David Halliday, Robert Resnick, and Jearl Walker, Fundamentals of Physics, 4th ed. (Wiley, New York, 1993), Essay 3.

${ }^{3}$ Douglas C. Giancoli, Physics for Scientists and Engineers, 2nd ed. (Prentice Hall, Englewood Cliffs, NJ, 1989), Chap. 13.

${ }^{4}$ Hans C. Ohanian, Physics (Norton, New York, 1989); Marcel Wellner, Elements of Physics (Plenum, New York, 1991); Marcelo Alonso and Edward J. Finn, Physics (Addison-Wesley, Reading, MA, 1992); Raymond A. Serway, Physics for Scientists and Engineers, 3rd ed. (Saunders College, Orlando, FL, 1992).

${ }^{5}$ Duncan J. Shaw, Introduction to Colloid and Surface Chemistry, 4th ed. (Butterworth-Heinemann, Oxford, 1992).

${ }^{6}$ Capillarity Today, edited by G. Pétré and A. Sandfeld, Lectures Notes in Physics No. 386 (Springer, Berlin, 1991); G. Cevc and D. Marsh, Phospholipid Bilayers: Physical Principles and Models (Wiley, New York, 1987).

${ }^{7}$ Francis W. Sears and Gerhard L. Salinger, Thermodynamics, Kinetic Theory and Statistical Mechanics, 3rd ed. (Addison-Wesley, Reading, MA, 1975); R. Becker, Theory of Heat (Springer, Berlin, 1967); O. Redlich, Thermodynamics: Fundamentals, Applications (Elsevier, Amsterdam, 1978).

${ }^{8}$ E. A. Guggenheim, Thermodynamics, 5th ed. (North Holland, Amsterdam, 1967).

${ }^{9}$ N. K. Adam, The Physics and Chemistry of Surfaces (Oxford University, Oxford, 1941).

${ }^{10}$ Samuel Glasstone, Textbook of Physical Chemistry (Van Nostrand, New York, 1949), Chap. 7.

${ }^{11}$ Francis W. Sears, Mark W. Zemansky, and Hugh D. Young, University Physics, 6th ed. (Addison-Wesley, Reading, MA, 1982), Chap. 12.

${ }^{12}$ A. W. Adamson, Physical Chemistry of Surfaces, 4th ed. (WileyInterscience, New York, 1982), Chap. 10.

${ }^{13}$ D. P. Woodruff, The Solid-Liquid Interface (Cambridge University, Cambridge, 1973), Chap. 2.

${ }^{14}$ David Tabor, Gases, Liquids and Solids and Other States of Matter, 3rd ed. (Cambridge University, Cambridge, 1993), Chaps. 6 and 10.
${ }^{15}$ Michael J. Jaycock and Geoffrey Derek Parfitt, Chemistry of Interfaces, 1st ed. rev. (Ellis Horwood, Chichester, 1986), Chap. 1.

${ }^{16} \mathrm{G}$. K. Batchelor, An Introduction to Fluid Dynamics (Cambridge University, Cambridge, 1987), Chap. 1.

${ }^{17}$ R. H. Fowler, Proc. R. Soc. London Ser. A 159, 229 (1937); Physica (Hauge) 5, 39 (1938), pointed out that the surface tension is not the internal energy nor the potential energy per unit area of the surface, but the superficial density of the Helmholtz free energy. It is interesting, however, to show here both physical interpretations.

${ }^{18}$ Some authors consider that there is no difference between the Helmholtz and the Gibbs function for this system. Even though the theory of generalized work (see, for example, Ref. 19) establishes that the Gibbs function of the interface should be defined as $G=F-\gamma A$, there is no definitive agreement yet. See Chap. 2 of Ref. 15 for a brief comparison of the two most common approaches.

${ }^{19}$ I. P. Bazarov, Thermodynamics (Pergamon, Oxford, 1964), Chap. 7.

${ }^{20}$ A. B. Pippard, Elements of Classical Thermodynamics for Advanced Students of Physics (Cambridge University, Cambridge, 1964), Chap. 6.

${ }^{21}$ Joseph W. Kane and Morton M. Sterheim, Physics (Wiley, New York, 1975), Chap. 8.

${ }^{22}$ A. Sandfeld, "Thermodynamics of surfaces," in Physical Chemistry. An Advanced Treatise, edited by (Academic, New York, 1971), Vol. I, Chap. 2C.

${ }^{23} \mathrm{~J}$. M. Haynes, "Surfaces," in Problems in Thermodynamics and Statistical Physics, edited by P. T. Landsberg (Pion, London, 1971), Chap. 8.

${ }^{24}$ Murray R. Spiegel and Lorenzo Abellanas, Mathematical Handbook of Formulas and Tables (McGraw-Hill, New York, 1970).

${ }^{25}$ Abraham Marmur, "Capillary rise in thin porous media," J. Phys. Chem. 93, 4873-4877 (1989).

${ }^{26}$ There are other mechanical approaches to explain capillary rise or depression [see, for example, Ernest K. Chapin, "Two contrasting theories of capillary action," Am. J. Phys. 27, 617-619 (1959)], but that in Fig. 4(c) is the most suited to this paper.

${ }^{27}$ H. N. V. Temperley and D. H. Trevena, Liquids and Their Properties (Ellis Horwood, Chichester, 1979), Chap. 9.

\title{
Vibrational properties of a loaded string
}

\author{
Samantha Parmley, Tom Zobrist, Terry Clough, Anthony Perez-Miller, Mark Makela, \\ and Roger Yu \\ Department of Physics, Central Washington University, Ellensburg, Washington 98926
}

(Received 6 September 1994; accepted 17 October 1994)

\begin{abstract}
In this paper we discuss our study of a string-mass chain and its anology to quantum mechanical systems. Theoretical predictions are made based upon the numerical solution to the wave equation. These predictions are tested experimentally using both normal mode analysis and pulse analysis. The frequency band structures for periodic and disordered string mass chains are studied as well as their corresponding eigenfunctions. The theoretical and experimental results are in accord. This experiment, suitable for advanced physics majors, demonstrates many important features of quantum mechanics: eigenvalues, superposition principle, band structure, gap modes, and Anderson localization. (C) 1995 American Association of Physics Teachers.
\end{abstract}

\section{INTRODUCTION}

The study of eigenstates and the corresponding eigenfunctions for periodic, quasiperiodic, and random systems has evolved from the Bloch periodic potential model to the Yablonovitch photonic crystal ${ }^{1}$ and to Maynard's quasiperiodic, macroscopic models. ${ }^{2,3}$ In this paper we present theo- retical models for periodic, quasiperiodic, and random string-mass chains and compare the theoretical model to our experimental results.

Periodic spring-mass and string-mass chains are extensively used as examples of eigenvalue problems because of the simplicity in mathematical treatment. For a string-mass chain, the mass of the string is usually assumed to be zero 
and the equation of motion of the $\boldsymbol{n}$ th mass for its transverse vibration of small amplitude yields the dispersion relation ${ }^{4}$

$$
\omega_{n}^{2}=\frac{4 T_{0}}{m a} \sin ^{2}\left(\frac{n \pi}{2(N+1)}\right), \quad n=1,2,3, \ldots, N-1 .
$$

Here $T_{0}$ is the tension, $m$ is the magnitude of the point mass, $a$ is the separation between masses, and $N$ is the number of masses. This dispersion provides a cutoff frequency, $\omega_{\max }=2\left(T_{0} / m a\right)^{1 / 2}$, which suppresses all the possible resonant frequencies above $\omega_{\max } \cdot{ }^{5,6} \mathrm{~A}$ more realistic model takes into account the mass of the string. The mass density function $\sigma(x)$ can be described by superimposing a delta function on a constant string-mass density. ${ }^{4}$ If the number of masses is sufficiently large, the Kronig-Penney model $(\mathrm{KP}),{ }^{7}$ in which an infinite string-mass chain is assumed, can be used to approximate the dispersion relation. Oseguera ${ }^{8}$ has employed the KP model and analytically shown multiple frequency bands and gaps in an infinite mass chain. Realistically, all the experimentally achievable samples are finite. For a finite sample, the vibrational localizations due to irregularity in a nearly periodic structure were studied experimentally and theoretically by Hodges and Woodhouse. ${ }^{9}$ Their theoretical method is based on the approach originally developed by Herbert and Jones ${ }^{10}$ and Thouless ${ }^{11}$ for a finite chain of $N$ pendula. The phenomenon of Anderson localization in an acoustic context was well demonstrated by the experiment reported in Ref. 9. More recently, a paper by Maynard and his co-worker ${ }^{2}$ describes a more refined experimental study on periodic and quasiperiodic string-mass systems. The authors found band structure and Anderson localization in the system by monitoring the transverse wave amplitude while sweeping the driving frequency.

For our model the theoretical predictions are made based upon the numerical solution of the wave equation. The differential wave equation is transformed into a matrix equation by Fourier expansion. With the formalism presented here it is possible to determine the eigenvalues and eigenfunctions to any degree of accuracy. There is no restriction on the distribution and the magnitude of the masses; therefore, the approach may be employed to simulate not only perfect onedimensional crystals, but also disordered crystals, quasicrystals, and amorphous structures.

On the experimental side, techniques of studying the dynamical properties of a microscopic electronic system or a macroscopic mechanical system fall into two categories: normal mode analysis (NMA) and pulse analysis (PA). In NMA, the transmitted amplitude through the system is recorded while the exciting (driving) frequency is being swept. The response of the system is expected to be enhanced when the exciting frequency is near a resonant (eigen)frequency. In $\mathrm{PA}$, a pulse, which is rich in frequency, is received by the system. The subsequent vibration of the system is recorded. Then the spectrum is obtained by carrying out a Fourier transform of the time series. The spectrum reveals all the important information about the eigenproperties of the system by peaking its value at eigenfrequencies. In the experiments reported here, both methods were used and the results are compared.

Another purpose of the present research project is to simulate quantum physics effects at a macroscopic level. In the classroom it is difficult to provide demonstrations of fascinating quantum mechanical phenomena. With our experi- mental setup, it is possible to achieve a classical wave system which precisely duplicates the salient features of quantum electronics.

\section{THE THEORETICAL MODEL}

The theoretical model was developed from the standard model for a vibrating string. The first deviation from the standard model accounts for variation in tension. The model is then expanded to include point masses. Due to the complexity of the resulting equation only the linear portion is solved for the massed string.

The nonlinear model developed accounts for the variation in tension due to amplitude of the wave form. This model has also been worked on by several other authors (e.g., Carrier, ${ }^{12}$ Lee, ${ }^{13}$ Oplinger, ${ }^{14}$ Murphy and Ramakrishna). ${ }^{15}$ With the assumption of vertical planar vibration one can find the change in tension as a function of position $x$ [Fig. 1(a)],

$$
T(x)=T_{0}+Y A(d s-d x) / d x,
$$

where $T_{0}$ is the tension at zero displacement, $Y$ is Young's modulus, $A$ is the cross-sectional area, and $d s$ is the stretched length of the element $d x . d s$ may be expressed in terms of the vertical displacement element $d u$, $d s=\left[(d x)^{2}+(d u)^{2}\right]^{1 / 2} ;$ then the tension becomes

$$
T(x)=T_{0}+Y A\left(\sqrt{1+\left(\frac{\partial u}{\partial x}\right)^{2}}-1\right),
$$

where the vertical displacement $u \equiv u(x, t)$. Now the equation of motion for the string, according to Newton's second law of motion, is ${ }^{15}$

$$
\sigma(x) \frac{\partial^{2} u}{\partial t^{2}}=\frac{\partial}{\partial x}[T(x) \sin (\theta)] .
$$

Here, $\sigma(x)$ is the line mass density of the string and

$$
\theta=\tan ^{-1}\left(\frac{\partial u}{\partial x}\right) \text {. }
$$

For small angle vibrations $\partial u / \partial x$ is much smaller than unity. Then, from Eq. (3) $T(x) \approx T_{0}+Y A / 2(\partial u / \partial x)^{2}$, and $\sin \theta$ $\approx \partial u / \partial x$. Equation (4), combined with these equations, may be reduced to

$$
\sigma(x) \frac{\partial^{2} u}{\partial t^{2}}=T_{0} \frac{\partial^{2} u}{\partial x^{2}}+\frac{3}{2} Y A\left(\frac{\partial u}{\partial x}\right)^{2} \frac{\partial^{2} u}{\partial x^{2}} .
$$

Since we are primarily interested in the eigenmodes of the system the solution $U_{n}(x, t)$ can be written as

$$
U_{n}(x, t)=U_{n}(x) \cos \left(W_{n} t\right),
$$

where the upper case $\Omega_{n}$ and $U_{n}(x)$ denote the nonlinear $n$th normal frequency and its wave function. Their linear counterparts are denoted by $\omega_{n}$ and $u_{n}(x)$. By substituting Eq. (7) into Eq. (6) and making the so-called "rotating-wave approximation" only a single frequency component is kept in the time dependence. ${ }^{15}$ Then Eq. (6) becomes

$$
\sigma(x) \Omega_{n}^{2} U_{n}+\left[T_{0}+9 Y A / 8\left(\dot{U}_{n}\right)^{2}\right] \ddot{U}_{n}=0 .
$$

We will first discuss the solution to this nonlinear equation for a bare string of length $L$ stretched with tension $T_{0}$. When the string vibrates in the vicinity of the $n$th eigenfrequency $\omega_{n}=n \pi / L\left(T_{0} / \sigma\right)^{1 / 2}$ predicted by the linear theory, the pattern of the standing wave can be approximated by the sine wave, 


$$
U_{n}(x)=b_{n} \sin (n \pi x / L),
$$

due to the boundary conditions; $u_{n}(0)=u_{n}(L)=0$. Substituting Eq. (9) into Eq. (8) and equating the coefficients of the $\sin (n \pi x / L)$ terms, the amplitude-frequency relation reads

$$
\sigma \Omega_{n}^{2}-T_{0}\left(\frac{n \pi}{L}\right)^{2}-\frac{9 Y A}{16} \beta_{n}^{2}\left(\frac{n \pi}{L}\right)^{4}=0,
$$

then,

$$
\Omega_{n}^{2}=\left(\omega_{n}\right)^{2}+\frac{9 Y A}{16 \sigma} \beta_{n}^{2}\left(\frac{n \pi}{L}\right)^{4} .
$$

It is clear that the resonant frequencies are shifted upward due to the nonlinearity of the string.

For a loaded string the mass density $\sigma(x)$ is no longer constant and the problem becomes rather complex. To our knowledge the equation has not yet been solved exactly. In the present paper, all the theoretical results are the solutions of the linear version of Eq. (6);

$$
\sigma(x) \frac{\partial^{2} u}{\partial t^{2}}=T_{0} \frac{\partial^{2} u}{\partial x^{2}} .
$$

Now, the linear version of Eq. (8) has the following form:

$$
\left(\omega_{n}\right)^{2} \sigma(x) u_{n}(x)+T_{0} \ddot{u}_{n}(x)=0 .
$$

We will numerically solve this equation and use perturbation theory ${ }^{4}$ to qualitatively determine the upshifted eigenfrequencies of the nonlinear system,

$$
\Omega_{n}^{2}=\omega_{n}^{2}+\int_{0}^{L} u_{n}(x) \beta_{n}^{2} \frac{9 Y A}{16}\left(\dot{u}_{n}\right)^{2} u_{n} d x .
$$

By taking advantage of the vanishing boundary conditions, the wave function in Eq. (13) can be expanded in a Fourier sine series,

$$
u_{n}(x)=\sqrt{2 / L} \sum_{m=1}^{\infty} c_{m}^{n} \sin \left(\frac{m \pi x}{L}\right) .
$$

An exact solution of Eq. (13) with any desired accuracy can be achieved by including a sufficiently large number of sine waves in the Fourier expansion. We have found that 100 sine waves, $N_{\max }=100$, lead to our desired accuracy. ${ }^{16}$

Upon substituting Eq. (15) into Eq. (13), multiplying both sides by $(2 / L)^{1 / 2} \sin (m \pi x / L)$, and integrating over $x$ from zero to $L$ the differential equation is transformed into the following $N_{\max } \times N_{\max }$ matrix equation: ${ }^{16}$

$$
\begin{aligned}
& \sum_{m=1}^{N_{\max }} c_{m}^{n}\left[\sigma_{l m} \omega_{n}^{2}-T_{0}\left(\frac{m \pi}{L}\right)^{2} \delta_{l, m}\right]=0, \\
& l=1,2, \ldots, N_{\max } .
\end{aligned}
$$

The matrix elements $\sigma_{l m}$ are defined as

$$
\sigma_{l m}=\int_{0}^{L} d x \sigma(x) \sin \left(\frac{l \pi x}{L}\right) \sin \left(\frac{m \pi x}{L}\right) .
$$

A software package in the IMSL Math/Library is used to diagonalize the matrix and to find the eigen frequencies $\omega_{n}$. The package also provides the coefficients of the wave functions [Eq. (15)] simultaneously.

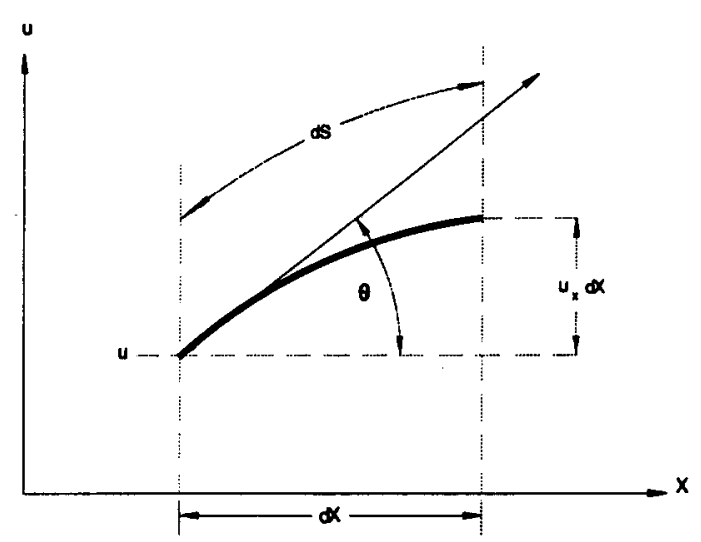

(a)

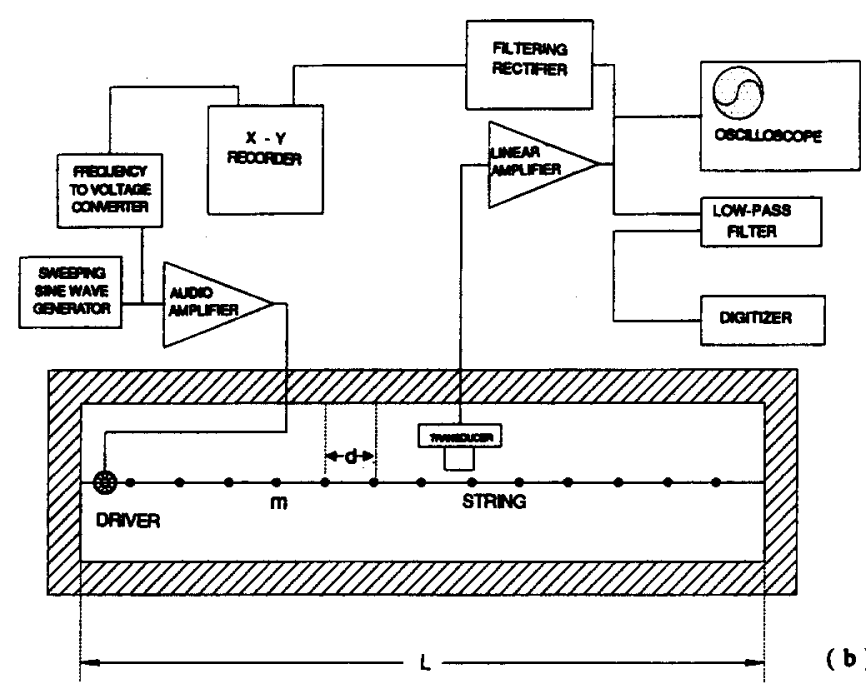

Fig. 1. (a) Illustration of the displacement of the string element $d x$. (b) The setup of the experiment on the loaded string. All the physical parameters are given in the text.

\section{EXPERIMENTAL METHODS AND APPARATUS}

As we discussed above, both techniques, NMA and PA, are used in studying the dynamical system. In reality, when NMA is used, the response of a nonlinear system depends not only on the driving frequency, but also on the driving amplitude. In general, upward frequency sweeping results in a different response from that of downward sweeping due to the hysteretic effect of a nonlinear system. In the present paper, all the NMA data are taken by sweeping the frequency upward. In PA, an external impulse, which is spectrally broad, excites the system to a transient state which is a linear superposition of its eigenstates. The time evolution of such a state in real space and its spectral density function in Fourier space reveal interesting physical properties of the system's resonance band structure.

The experimental setup to drive and record the motion of the string is schematically shown by the flow chart in Fig. 1(b). A Tektronix FG 504 Sweeping Function Generator, which can either be set to specific frequencies or to sweep through a range of frequencies, produces a sinusoidal wave. The signal is then run through an amplifier to an audio 


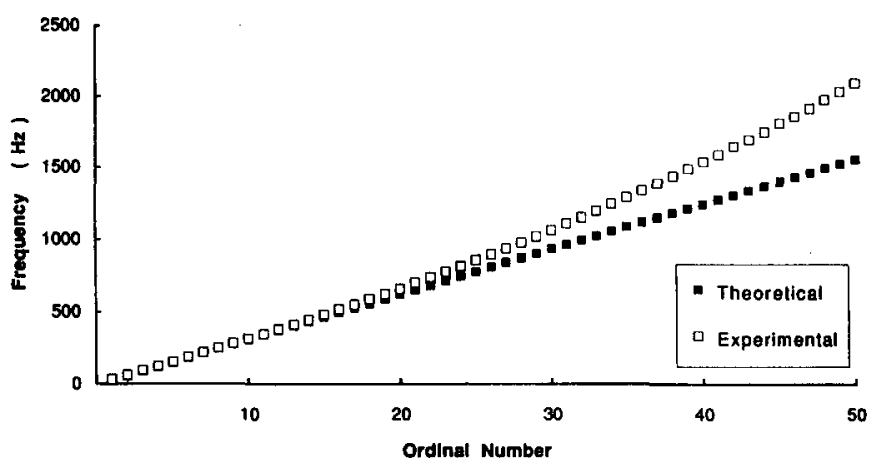

Fig. 2. Resonant frequencies of a bare string vs. the ordinal number.

speaker. The string is physically attached to the voice coil of the speaker so that the string and the speaker vibrate at the same frequency.

We have devised two methods for recording data from the transducer. One is to run the signal from the transducer through a rectifier to produce a dc signal which corresponds to the string's amplitude. This signal is then run into a Sargent-Welch $x-y$ recorder which produces a hard copy graph of amplitude versus frequency for the range of frequencies swept by the function generator. This method allows us to perform NMA. The second method of recording the data is based on the use of a computer driven digitizer. The signal from the transducer is run through a linear amplifier, then through a low-pass filter and into the digitizer. The low-pass filter is used to eliminate aliasing. The digitized data enable us to analyze the real time series in the frequency domain by fast Fourier transform (FFT). The spectrum exhibits peaks which can be used to identify the eigenfrequencies of the system. This method is used primarily for PA and for looking at the spectral component for a given eigenfrequency.

The physical parameters of the system used in the experiment shown in Fig. 1(b) are the following: string length $L$ is $1.65 \mathrm{~m}$, mass density is $3.35 \times 10^{-3} \mathrm{~kg} / \mathrm{m}$, each mass is 0.49 $\mathrm{g}$, tension is $36.7 \mathrm{~N}$. In all the calculations reported here, we have assumed that the change in tension due to adding the masses is negligible, which is a good approximation because the total mass loaded is only about $5 \mathrm{~g}$.

\section{RESULTS AND DISCUSSION}

In this section we present our experimental results and discuss how they compare to our theoretical model. We first look at normal mode analysis for the bare string and string with up to five masses. Then we look at pulse analysis for the same system. And finally we discuss the effects of disordered mass spacing.

The experimental data gathered from the bare string diverged from the linear model which caused us to look at the nonlinear model; this divergence is shown in Fig. 2. The divergence appears to be parabolic, this can be explained as a combination of the change in amplitude and the nonlinearity of the system. As the string is stretched vertically the tension increases and so does the natural frequency; however, the amplitude of vibration decreases with frequency as $1 / n^{1 / 2}$. In addition, the solution of the nonlinear model, Eq. (11), indicates that the upshift in frequency squared is proportional to $n^{4}$, while $B_{n}$ decreases as $1 / n^{1 / 2}$. Taking these

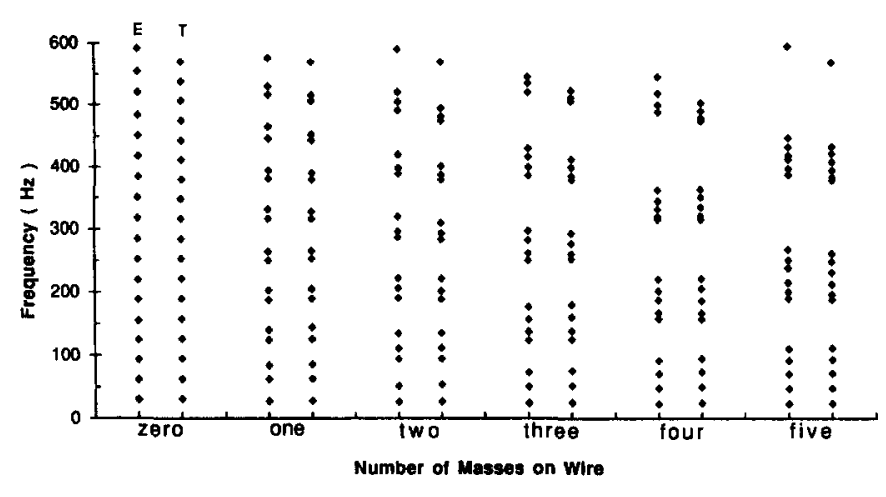

Fig. 3. Resonant frequencies of a loaded string. For the string with fixed number of masses, the calculated (experimental) data are plotted in the right-hand (left-hand) column.

two factors into consideration, the nonlinear solution has a parabolic shift which agrees with our experimental data.

Figure 3 depicts the theoretical (right-hand column) and the experimental (left-hand column) resonant frequencies measured by the NMA for up to five point masses. The calculated frequencies are obtained by solving the wave equation [Eq. (16)] for each massed system. For comparison, we also show the bare string frequencies. For a string with one mass at the center, there would be only one resonant frequency if the mass of the string were neglected. ${ }^{4,8}$ In the present case, resonant frequencies above the lowest one are found since the mass of the string is finite. The measured frequency of the lowest mode $(n=1)$ is slightly lower than that of the no-mass string since the string feels the inertia of the point mass. For the second mode, the mass is stationary and only the string moves; this is why its frequency coincides with the second of the no-mass string. In the third mode, the mass vibrates again, but its amplitude is suppressed and so is its frequency. The point mass is stationary for $n=4$, whose frequency again matches that of the no-mass string. As one can see from Fig. 3, for the one-mass string, the frequencies are paired, except the lowest mode. For the two-mass string, the lowest two eigenmodes, describing the in-phase and out-of-phase motions of the masses, form the lowest frequency band and the upper bands are composed of three eigenmodes in which the point masses are almost stationary. The formation of frequency bands is even more clear as the number of masses becomes larger. Figure 3 (e.g., five mass data) shows that the widths of higher bands become narrower. This is due to the greater suppression of motion of the point masses. It is also seen that the width of a certain band increases as the number of loaded masses increases. If the separation between the masses is kept constant, the frequency bandwidth ought to be saturated as the number of masses becomes large. However, in the experiment performed here, the total length of the string is constant, and the mass separation becomes smaller with more evenly loaded masses; therefore, the bandwidth increases accordingly. It is also worth noting that, in general, the experimental frequencies are higher than the theoretical ones predicted by linear theory. The discrepancy becomes larger for higher modes. Based on perturbation theory [Eq. (14)], the shift in frequency squared due to the nonlinearity depends upon the integration. In the integrand, $b_{n}^{2}$ decreases in $n$, but the square of $d u_{n}(x) / d x$ increases faster than $n^{2}$ when the string is loaded with masses. Therefore the frequency shift becomes larger at higher modes. 


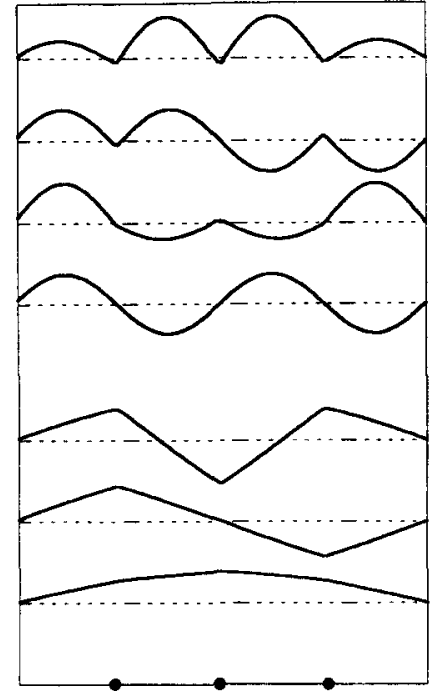

Fig. 4. The lowest seven eigenfunctions of the 3-mass string. The positions of masses are marked. The lowest three eigenstates, forming the lowest band, show much greater vibration amplitude of masses.

To better understand the eigenmodes and the formation of the bands, we plotted the calculated lowest seven eigenfunctions [see Eq. (13)] in Fig. 4, for the three-point-mass system. These seven eigenmodes constitute the lowest two bands, as shown in Fig. 3. For the fundamental and the first two harmonics, the masses are at antinodes; whereas, in the first excited band the string segments have much larger amplitude than the masses.

The eigenmodes of the string are studied using PA by plucking the string and recording its time series, $G(x, t)$, at a chosen position $x=a$. The frequency spectrum, $S(\omega)$, is obtained from the time series by FFT.

The time series and the frequency spectrum for the bare string plucked near one end seem to be in good accord with the NMA data. The time series, shown in Fig. 5(a) indeed seems noisy; however, the FFT [Fig. 5(b)] clearly shows strong resonance peaks. At lower frequencies the peaks are almost evenly spaced, whereas at higher frequencies the spacing between neighboring peaks increases as also shown in Figs. 2 and 3. If the theoretical results are compared, one finds that the odd modes, whose wave forms are antisymmetric, have relatively much weaker oscillatory strength (see the weak structure in between two strong peaks). We believe this is because the initial displacement is an even function. We also note that the oscillator strength favors lower frequency modes whose wavelengths are more comparable with that of the initial displacement.

The time series for the three-mass string looks similar to that for the bare string; however, its FFT shows that the frequency components are grouped (Fig. 6). The spectrum is easier to understand if one compares it with Fig. 3. Three peaks near 30,50 , and $80 \mathrm{~Hz}$ form the lowest band. The excited bands are all composed of four resonant states as seen in Fig. 3. In all the excited bands, the lowest two resonant frequencies are closer to each other. This trend is also displayed in both Figs. 3 and 6 . The band gap becomes wider at higher frequencies this is also consistent with Fig. 3. Quantitatively, the NMA and PA data match very well.

In order to study periodicity and disorder we use a string with more point masses. We expect that the difference between a perfectly periodic and a random system would be
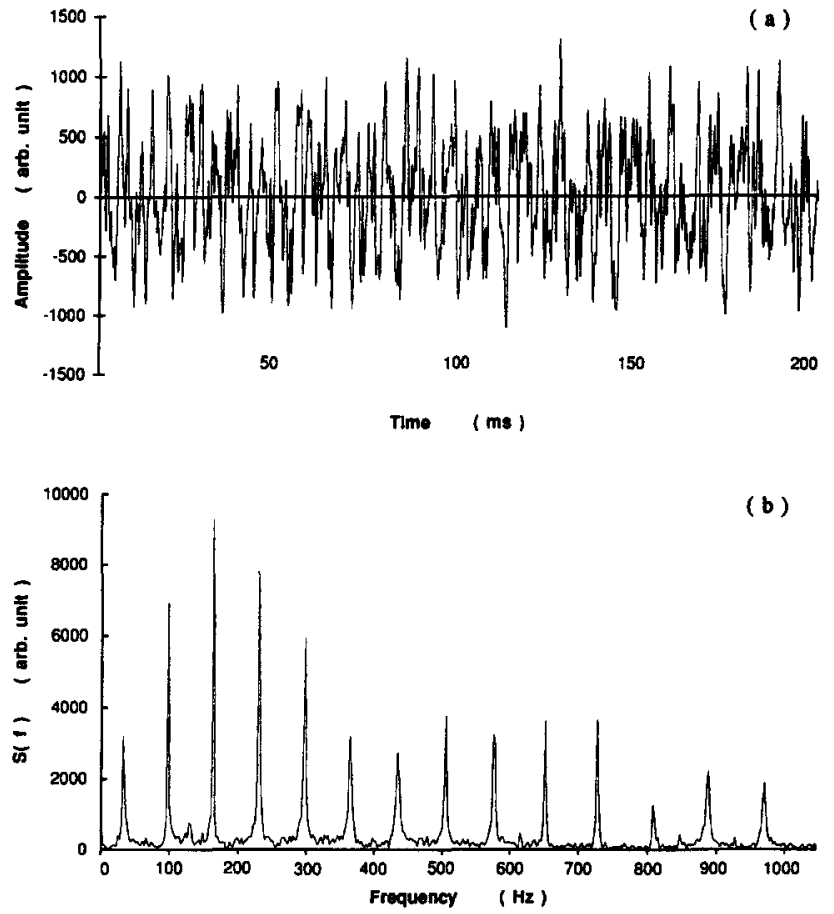

Fig. 5. The experimental vibration signal (a) and its Fourier transform (b) for the bare string. The response of the odd eigenmodes $(n=2,4,6, \ldots)$, whose eigenfunctions are antisymmetric, are missing due to the fact that the plucked string form is closer to an even function. The frequency spacing increases slightly because of the nonlinearity of the string.

more significant in a string with more masses. We present the results of ordered and disordered systems in a parallel fashion; figures for different systems are shown together for comparison. Thirteen masses are evenly spaced on the string
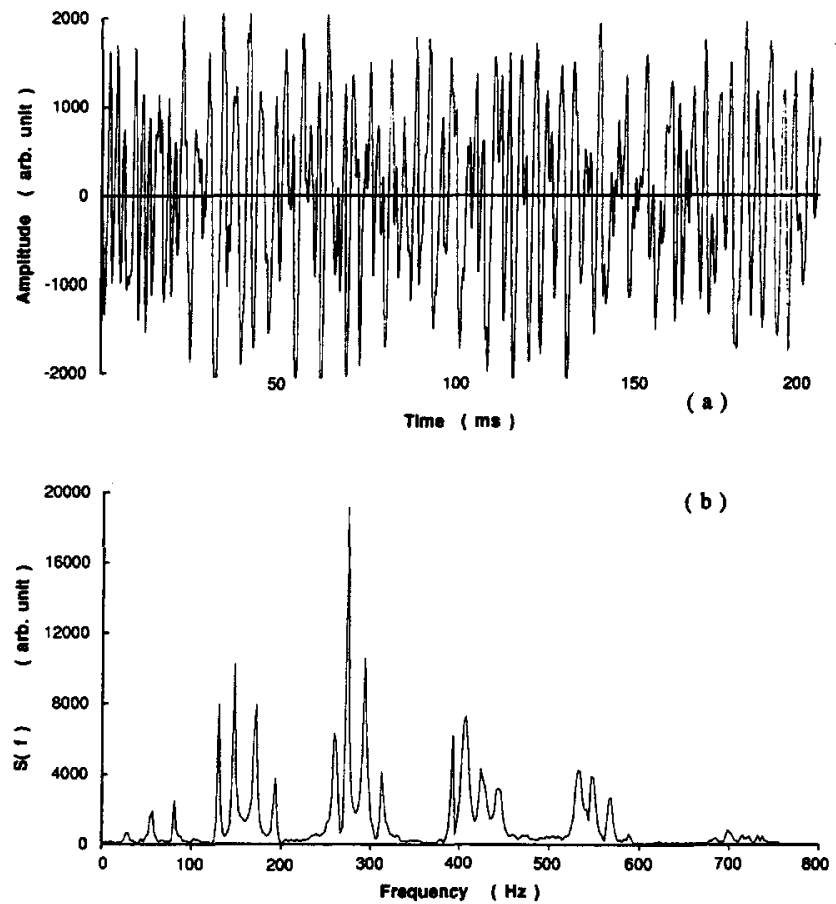

Fig. 6. The experimental vibration signal (a) and its Fourier transform (b) for the 3-mass string. The localizations of peaks closely match the result shown in Fig. 3. 

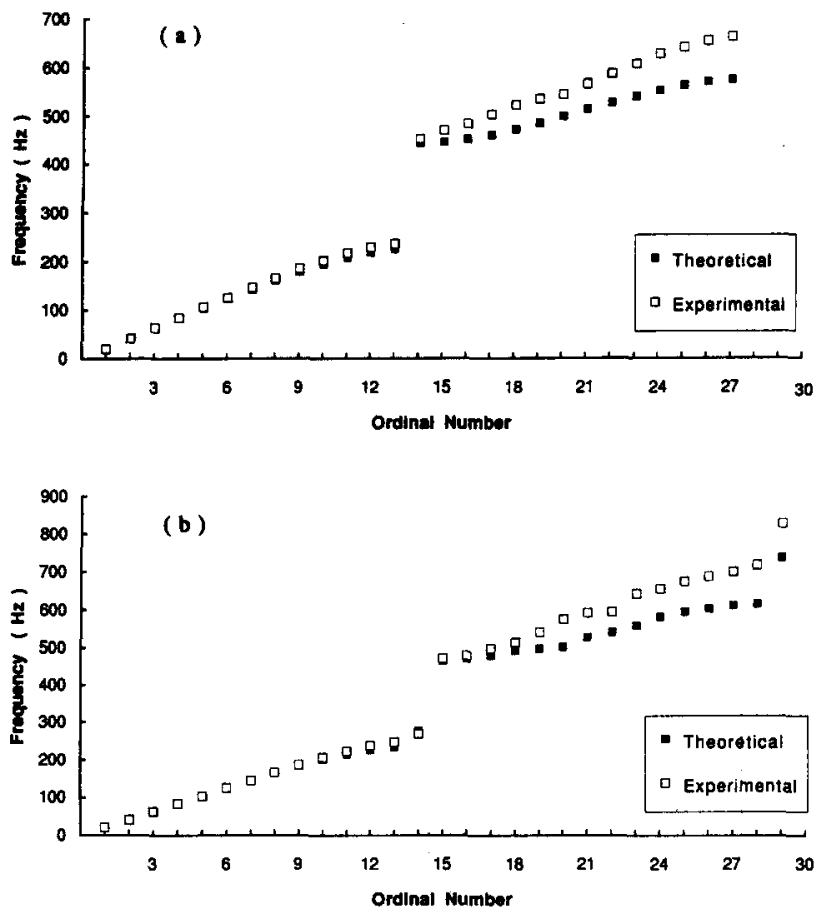

Fig. 7. a (b) shows the resonant frequencies of the periodic (random) 13mass string as a function of the ordinal number $n$.

for the periodic system. Random variation in mass separation is incorporated in the disordered string. It can be seen in Fig. 7 that the NMA data and the theoretical predictions match quite well for both systems, especially in the lowest bands. The dispersion is especially interesting in two limits; long wavelength and short wavelength. At the Brillouin zone boundary, $n=13$, the group velocity of the system, which equals the derivative of frequency with respect to the wave vector $k$ (or $n$ ), is almost zero. It is clearly seen in the eigenfunctions shown in Fig. 8(a) that when $n=13$, all the masses are vibrating out of phase and the wavelength $\lambda=2 a$, so that the wave of the system looks more like a standing wave with zero group velocity. At the other end of the Brillouin zone boundary, $n=1$, the wavelength $\lambda$ is large. One can view the system as a continuum and the string wave becomes an acoustic wave. ${ }^{17}$ In the second band, significant discrepancy between the theoretical and experimental data is observed. The experimental data shows a nonzero group velocity (finite slope) near $n=14$. The structure in the middle of the band, $n=20$, displays a smaller group velocity. Maynard and his co-workers ${ }^{2}$ have observed regions of low response in the second band in a similar measurement. They attributed the effect to the $\sim 13 \%$ variation in the size of the commercial lead masses. We believe that the same argument may be applied to our data shown in Fig. 7.

The dispersion relation for the random system, shown in Fig. 7(b) resembles that of the periodic system in the lowest band. The wave functions of the lowest band for both systems are all extended (see Fig. 8). In a disordered system, the wave functions generally have three types of characteristics: (i) extended, (ii) localized, and (iii) critical which is the combination of (i) and (ii). ${ }^{18}$ In Fig. 8(b), the gap mode (GAP 1) is strongly localized whereas the lowest and the highest states in the second passing band $(n=14,27)$ are critical. The gap mode in between the second and third bands (GAP 2) is also highly localized. ${ }^{19}$

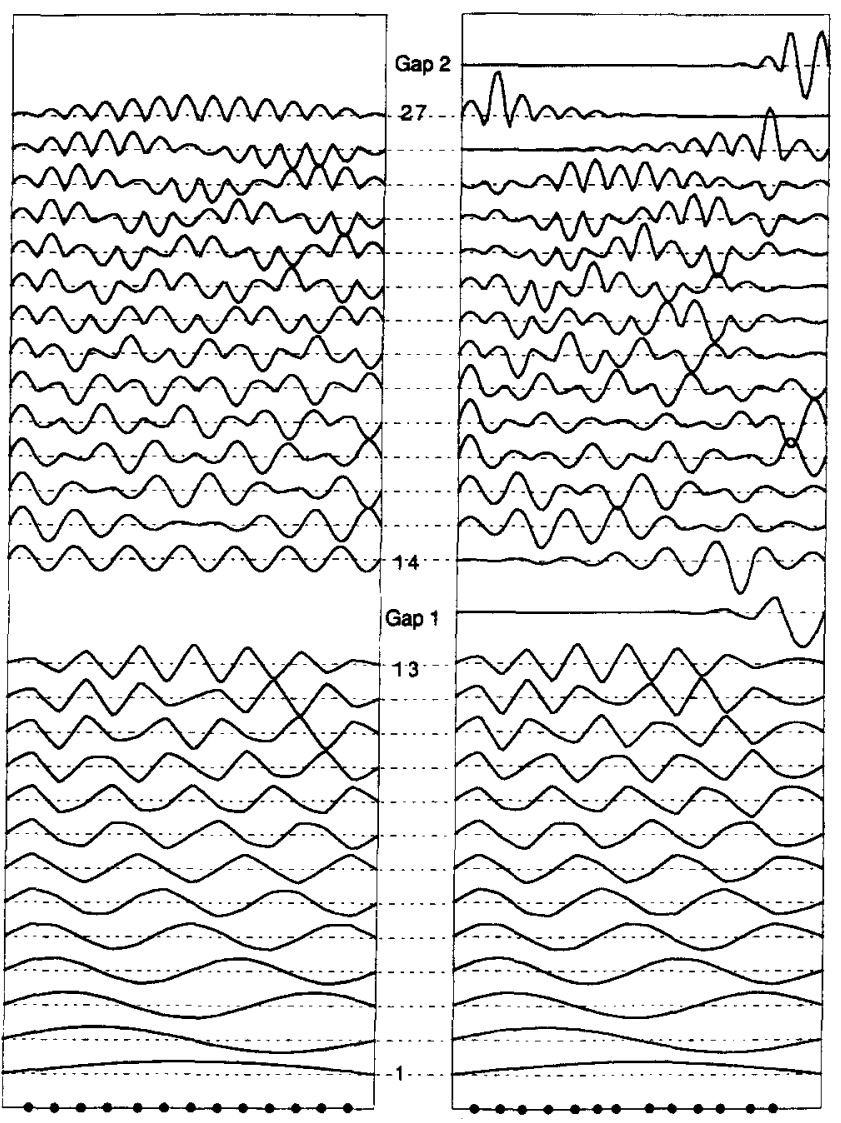

Fig. 8. The eigenfunctions of the lowest two bands of the periodic (left-hand column) and the random (right-hand column) 13-mass string. The positions of masses are marked.

In the spectrum $S(\omega)$, [Fig. 9(a)], four frequency bands are distinguishable for the periodic system. In the first and second band, one can actually see 13 and 14 peaks, respectively, corresponding to 13 and 14 eigenfrequencies in each band. The first band has a more powerful frequency spectrum. Again, the size of the plucked initial wave form is in the same order of magnitude as wavelengths of the lowest band. One of the theoretical (linear theory) predictions for the mechanical system considered here is that the bandwidth of higher bands decreases, which is just the opposite of an electronic band structure. This is due to weaker coupling between string segments caused by lower amplitude vibration of the masses. The theoretical wave forms in Fig. 8(a), show that the amplitude of vibration of the masses becomes smaller in the higher bands. In contrast, the measured frequency bandwidth does not decrease significantly because of the upshift in frequency due to the nonlinearity of the system.

For the disordered system, three bands are visible in the spectrum shown in Fig. 9(b). The distinct feature in the spectrum is the active response in the gaps. Immediately above the lowest passing band and below the second band, weak peaks corresponding to the localized gap mode can be easily observed.

In summary, we have studied the vibrational properties of a string loaded with point masses, both numerically and experimentally. For a periodically loaded string we have found that the resonant frequencies form bands whose wave functions are extended. In contrast to the periodic system, strongly localized modes (in the gaps or near the edges of the 

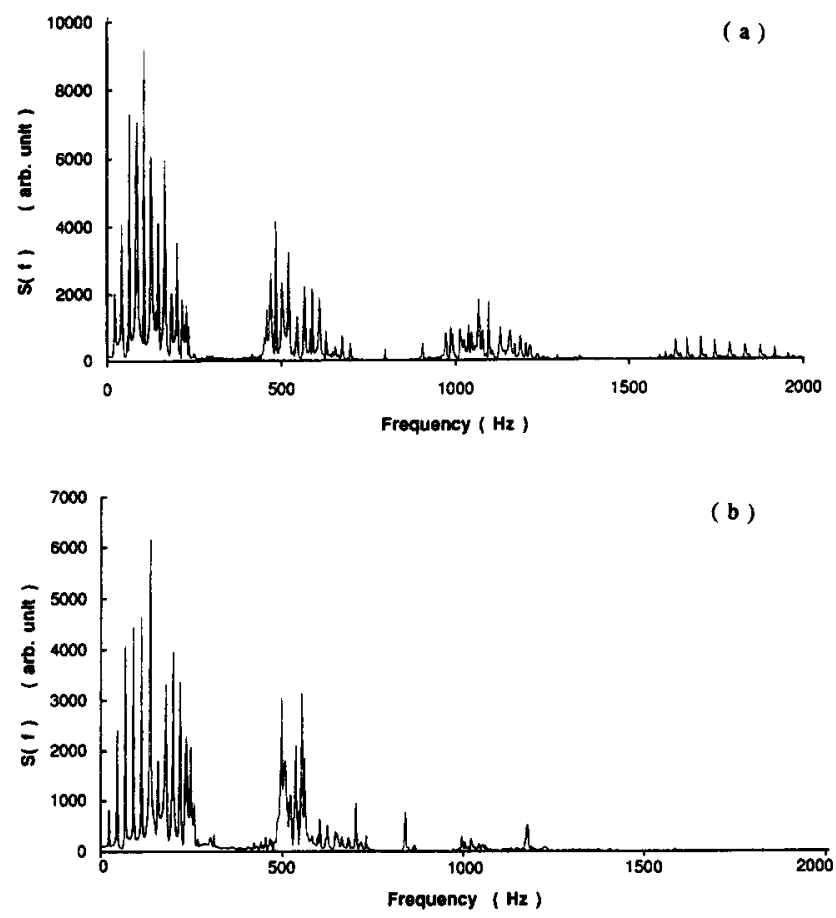

Fig. 9. Spectrum for the periodic (a) and random (b) 13-mass string.

frequency bands) are found in a randomly loaded string. The physical properties found in the classical system are reminiscent of many important quantum mechanical effects. The experiment presented here provides vivid visualization of those effects in our advanced physics courses.

\section{ACKNOWLEDGMENTS}

One of the authors (S.P.) appreciates the support from the Council on Undergraduate Research Summer Opportunities for Research (CURSOR) Fellowship. R. Y. thanks the College of Graduate Studies and Research at Central Washington University (CWU) for financial support, and the Department of Computing and Telecommunication at CWU for technical support.
${ }^{1}$ E. Yablonovitch, J. J. Gmitter, and K. M. Leung, "Photonic Band Structure: The Face-Centered-Cubic Case Employing Nonspherical Atoms," Phys. Rev. Lett. 67, 2295-2298 (1991).

${ }^{2} \mathrm{~S}$. He and J. Maynard, "Detailed Measurement of Inelastic Scattering in Anderson Localization," Phys. Rev. Lett. 57, 3171-3174 (1986).

${ }^{3} \mathrm{~S}$. He and J. Maynard, "Eigenvalue Spectrum, Density of States, and Eigenfunctions in a Two-Dimensional Quasicrystal," Phys. Rev. Lett. 62, 1888-1891 (1989).

${ }^{4}$ A. L. Fetter and J. D. Walecka, Theoretical Mechanics of Particles and Continua (McGraw-Hill, New York, 1980).

${ }^{5}$ G. Shanker, v. Gopta, and N. Sharma, "Normal Modes and Dispersion Relations in a Beaded String: An Experiment for an Undergraduate Laboratory," Am. J. Phys. 53, 479-481 (1985).

${ }^{6} \mathrm{M}$. Siva et al., "Spring-Mass Chains: Theoretical and Experimental Studies," Am. J. Phys. 58, 923-928 (1990).

${ }^{7}$ R. Kronig and W. Penney, "Quantum Mechanics of Electrons in Crystal Lattices," Proc. R. Soc. London Ser. A 130, 499-512 (1931).

${ }^{8}$ U. Oseguera, "Classical Kronig-Penney Model," Am. J. Phys. 60, 127130 (1992).

${ }^{9} \mathrm{C}$. Hodges and J. Woodhouse, "Vibration Isolation from Irregularity in a Nearly Periodic Structure: Theory and Measurements," J. Acoust. Am. 74, 894-905 (1983).

${ }^{10}$ D. C. Herbert and R. Jones, "Localized States in Disordered Systems," J. Phys. C: Solid State Phys. 4, 1145-1161 (1971).

${ }^{11} \mathrm{D}$. Thouless, "Localization Distance and Mean Free Path in OneDimensional Disordered Systems," J. Phys. C: Solid State Phys. 6, L49_ L51 (1973).

${ }^{12}$ G. Carrier, "On the Nonlinear Vibration Problem of the Elastic String," Q. Appl. Math. 3, 157-165 (1945).

${ }^{13} \mathrm{E}$. Lee, "Non-linear Forced Vibration of a Stretched String," Brit. J. Appl. Phys. 8, 411-413 (1957).

${ }^{14} \mathrm{D}$. Oplinger, "Frequency Response of a Nonlinear Stretched String," J. Acoust. Soc. Am. 32, 1529-1538 (1960).

${ }^{15} \mathrm{G}$. Murphy and B. Ramakrishna, "Nonlinear Character of Resonance in Stretched String," J. Acoust. Soc. Am. 38, 461-471 (1965).

${ }^{16}$ Roger Yu, "Self-Consistent Determination of Electronic Structure and Elementary Excitations of Finite Modulation-Doped Superlattice," Phys. Rev. B 47, 15692-15699 (1993); William Glessner and Roger Yu, "Novel Surface Electronic State in Finite Superlattice," J. Appl. Phys. 73(8), 4070-4071 (1994).

${ }^{17}$ G. P. Srivastava, The Physics of Phonons (Hilger, London, 1990).

${ }^{18} \mathrm{~J}$. Lu, T. Odagaki, and J. Birman, "Properties of One-Dimensional Quasilattices," Phys. Rev. B 33, 4809-4817 (1986).

${ }^{19} \mathrm{P}$. Anderson, "Absence of Diffusion in Certain Random Lattices," Phys. Rev. 109, 1492-1505 (1958).

\section{HOMEWORK PROBLEMS}

I started my attempt. Step 1. Draw picture.

What would give you uniform heat flux anyway? If the heat flux is uniform, why isn't the temperature uniform? Ack-ack, as Bill the Cat would say.

Step 2. Look for similar sample problem in book. There isn't one that's even close.

Step 3. Look through four different heat transfer books on office mate's shelf. Rohsenow said yesterday that we could bring to the test any book we wanted to. "The only thing you can't bring," he said with a wink, "is a consultant." There's nothing in them, either.

Step 4. Look through class notes, then office mate's class notes from previous year's lecture. Another dead end.

Step 5. Think about what's going on in the problem. Refer to the picture. Redraw the picture.

Pepper White, The Idea Factory-Learning to Think at MIT (Penguin Books, New York, 1991), pp. 40-41. 